\title{
Influences of Vitamin D and B12 in Infectious Diseases and Microbes: A Technical Review
}

\author{
Jase Grimm \\ University of the People, 595 E Colorado Blvd Suite 623, Pasadena, CA 91101, USA
}

This journal is licensed under a Creative Commons Attribution-Noncommercial 4.0 International License (CC-BY-NC).

Articles can be read and shared for noncommercial purposes under the following conditions:

- BY: Attribution must be given to the original source (Attribution)

- NC: Works may not be used for commercial purposes (Noncommercial)

This license lets others remix, tweak, and build upon your work non-commercially, and although their new works must also acknowledge you and be non-commercial, they don't have to license their derivative works on the same terms.

License Deed Link: http://creativecommons.org/licenses/by-nc/4.0/

Legal Code Link: http://creativecommons.org/licenses/by-nc/4.0/legalcode

$A B C$ Research Alert uses the CC BY-NC to protect the author's work from misuse.

\section{Abstract}

A diet rich in vitamin D. Bacteria, antibiotics, and metabolism Vitamin D deficiency viral microbiomes Gut Microbiome and Vitamin D. B vitamin deficiency results from malabsorption. SIF complex indirectly enhances B12 absorption. This complex gives the ileum vitamin B12. Anti-infective properties of B12 and D. B12 and D seem to slow Lachnospiraceae growth. P-cresol, butyrate, propionate Lachnospira is linked to low B12 and D levels. Debatable value. Processed foods do. B12 and D stop Blautia growth. IBD B12 deficiency IBD vitamin D Lactobacillus was infrequent, whereas E. coli was common. This present study revealed that IPA, an antioxidant, has been associated with diabetes and Alzheimer's. IPA beats melatonin. It doesn't appear to stop Alzheimer's or diabetes. Flavonoids and neuroprotective viruses D and B12 metabolism may involve Blautia. Deficiency of B12 and D in Crohn's. It reduced butyrate production. Inflammation, atherosclerosis, and immunological maturation may be cured by these nutrients.

\section{Keywords}

Vitamin D, Vitamin B12, Helicobacter pylori, Fungi, Tuberculosis.

\section{INTRODUCTION}

Vitamin D and B12 are essential nutrients that humans derived from both diet and in the case of vitamin D sunlight. Besides metabolic processes these vitamins also influence infectious diseases and microorganisms of various types which is discussed in this review of the literature.

Although not the subject of this review, it should be noted that viral microbiomes are also associated with lowered vitamin D. For general microbiome developments this review limits itself to studies during and after 2019 as a systematic review titled "Vitamin D and the gut microbiome: a systematic review of in vivo studies." was published in October 2019, which covers the prior general gut microbiome literature (Waterhouse, Mary, et al.).

Vitamin B12 is one of eight B vitamins, which are only available to humans through diet and as such malabsorption is a common route to deficiency (Figure 1). The stomach plays an important, although indirect, role in vitamin B12 absorption, by producing the intrinsic factor complex. Vitamin B12 transitions over the course of digestion from food proteins to the intrinsic factor complex and is primarily absorbed in the ileum of the small intestine by enterocytes. 


\section{METHODS}

PubMed was searched using the following terms:

Query = "vitamin b 12"[MeSH Terms] AND ("infections"[MeSH Terms] OR "infections"[All Fields] OR "infection"[All Fields]) AND 1990/01/01[EDAT] : 2021/08/16[EDAT]

Query = ("vitamin d"[MeSH Terms] OR "ergocalciferols"[MeSH Terms]) AND ("infections"[MeSH Terms] OR "infections"[All Fields] OR "infection"[All Fields]) AND 1990/01/01[EDAT]: 2021/08/16[EDAT]

The returned papers were then reviewed and interpreted as presented in the following sections.

\section{RESULTS}

\section{Helicobacter pylori}

A well-studied chronic bacterial pathogen of the stomach called Helicobacter pylori can cause stomach ulcers and also has a well-established negative relationship with serum vitamin B12 levels under most circumstances, although there is some evidence that in certain populations such as the obese and those with chronic kidney disease that $\mathrm{H}$. pylori infection can actually increase serum vitamin B12 levels.

H. pylori has a newly established inverse relationship with serum vitamin D levels under most circumstances, particularly eradication therapy. In a large Isreali study the odds of a positive $\mathrm{H}$. pylori test were $31 \%$ higher (OR 1.31, 99\% CI 1.22-1.4, p < 0.001) among patients with deficient vitamin D levels $(<20 \mathrm{ng} / \mathrm{mL})$ (Shafrir, Asher, et al.). In a fully controlled mouse study vitamin D was demonstrated to have a mechanism of action against $H$. pylori by acting through the vitamin $\mathrm{D}$ receptor (VDR) and the cathelicidin antimicrobial peptide (CAMP) (Zhou, Anni, et al.). As with all microbes, drug resistance in $\mathrm{H}$. pylori is a growing problem. The Chinese University of Hong Kong began a phase 3 clinical trial (NCT03142620) in 2017 to test the hypothesis that vitamin D in combination with standard antimicrobial therapeutics could improve the eradication rates of drug-resistant $H$. pylori. Results are pending.

There is also some evidence that in certain populations such as those with chronic kidney disease and possibly when vitamin $\mathrm{D}$ is obtained through oily fish that $H$. pylori infection is associated with increased serum vitamin D levels (Nasri, Hamid, and Azar Baradaran.), (Ikezaki, Hiroaki, et al.). The reasons for these exceptions are not clear as there are possibly confounding factors.

\section{Gut Bacterial Microbiome}

Certain intestinal bacteria can synthesize and or sequester vitamin B12. For this reason the gut microbiome can play an important role in the availability of vitamin B12. Studies on the effects of probiotics on serum vitamin B12 status are limited, however it was found that Pediococcus acidilactici and Saccharomyces cerevisiae boulardii had no effect on serum vitamin b12 levels in the short-term (Nadeau É., et al.). These types of studies are complicated by the fact that the liver stores large reserves of vitamin B12, which can buffer short-term deficiencies. This suggests that microbiome changes must be chronic in order to bring about serum vitamin B12 changes.

In pigs infected with Lawsonia intracellularis, the causative agent of porcine proliferative enteropathy, only the chronic version of the disease called porcine intestinal adenomatosis, (PIA), not the acute proliferative hemorrhagic enteropathy (PHE), was associated with vitamin B12 deficiency (Grützner, Niels, et al.).

Vitamin D had no effect on Lawsonia intracellularis; about sixty-six percent of pigs were positive regardless of vitamin D status.

The "good" bacterium Bacteroides thetaiotaomicron can inhibit Shiga toxin 2 (Stx2) production by Enterohemorrhagic Escherichia coli (EHEC) via poorly defined soluble factors (Cordonnier, Charlotte, 
et al.). Importantly, a B. thetaiotaomicron clone with mutant BtuB, an outer membrane receptor for vitamin B12, was unable to inhibit Stx2 production. In this context there appears to be a balance of power between EHEC and B. thetaiotaomicron, which can be shifted depending on the availability of vitamin B12.

Interestingly, prebiotics can stimulate the synthesis of the vitamin D precursor 7-dehydrocholesterol in Bacteroides thetaiotaomicron (Gokhale, Sucheta, and Anirban Bhaduri.). Therefore, a diet containing vitamin B12 and prebiotics can regulate the ability of the gut microbiome to inhibit pathogenic bacteria and to synthesize vitamin D precursors. Vitamin D metabolism is therefore dependent on vitamin B12 to a certain extent, at least at the level of the gut microbiome.

The relationship between vitamin D and the gut microbiome is complicated by the existence of at least three distinct enterotypes in people (Arumugam, Manimozhiyan, et al.), however there may be stable associations between the presence of certain microbiome community members and the level of serum vitamin D. Prevotella were more abundant $(\log 2 \mathrm{FC} 1.67, \mathrm{p}<0.01)$, while Haemophilus $(\log 2 \mathrm{FC}-2.92$, $\mathrm{p}<0.01)$, Veillonella $(\log 2 \mathrm{FC}-1.46, \mathrm{p}<0.01)$, Coprococcus $(\mathrm{r}=-0.215, \quad \mathrm{p}=0.008)$ and Bifidobacterium ( $\mathrm{r}=-0.269, \mathrm{p}=0.001$ ) were inversely correlated with $25(\mathrm{OH}) \mathrm{D}$ (Luthold, Renata V., et al.). Inflammation appears to regulate this relationship but exactly how is not yet clear. In mice that had been engineered so that they could not synthesize vitamin D or its receptor, an altered composition of the gut microbiome resulted consistent with the human results correlating vitamin D levels with microbiome composition (Ooi, Jot Hui, et al.), (Jin, Dapeng, et al.).

In a genome-wide association (GWAS) study, human vitamin D receptor (VDR) polymorphisms consistently affected Parabacteroides of phylum Bacteroidetes. Further investigation in VDR-/- mice showed an increase in Parabacteroides compared to wild type mice (Wang, Jun, et al.). Conversely, in a study involving mice induced to be allergic to food and then treated using phototherapy it was found that Parabacteroides goldsteinii increased and Lachnospiraceae representation of the phylum Firmicutes decreased (Chen, Po-Jung, et al.). Consistent with this, in healthy Japanese monozygotic twins, Lachnospira had a significantly negative correlation with protein, sodium, iron, vitamin D, vitamin B6, and vitamin B12 intake ( $\mathrm{rs}=-0.38,-0.41,-0.39,-0.63,-0.42,-0.49$, respectively) (Matsumoto, Natsuko, et al.). Given that vitamin D and vitamin B12 had the two biggest negative effects on Lachnospira individually, it would be interesting to know whether the two vitamins have an additive effect in combination.

In people high vitamin D intake has also been negatively associated with Prevotella and strongly positively associated with Bacteroides, both of the phylum Bacteroidetes (Wu, G. D., et al.). On the other hand some studies have found no associations between vitamin D intake and the gut microbiome (Shieh, Albert, et al.).

In infants from birth to four months a positive correlation was found between the level of serum vitamin D and the abundance of Bifidobacterium.

Vitamin D supplementation may lower the risk of atherosclerosis, type I diabetes, and intestinal cancer by acting upon the gut microbiome, particularly promoting the growth of the genus Akkermansia, Butyricimonas and other Odoribacteraceae family members (Wang, Xin, et al.), (Rabbone, Ivana, et al.), (Robles-Vera, Iñaki, et al.), (Zhou, Xueyan, et al.), (Zhang, Yong-Guo, et al.), (Charoenngam, Nipith, et al.). These effects may be context dependent as not all studies find a significant influence on Akkermansia (Chatterjee, Ishita, et al.).

In the northern hemisphere serum vitamin D levels fluctuate with the season as do the microbiomes of the Inflammatory bowel diseases (IBDs) Crohn's disease (CD) and ulcerative colitis (UC) (Soltys, Katarina, et al.).

Interestingly, it appears that immunodeficiency nullifies vitamin D's ability to modulate the gut microbiome as HIV-1 patients supplemented with 5000 IU vitamin D did not demonstrate any significant change in their microbiomes (Missailidis, Catharina, et al.). 
Furthermore, dysbiosis due to vitamin D deficiency may induce a negative feedback loop in which the accumulating dysbiotic bacteria actively limit vitamin D biosynthesis (Zuo, Kun, et al.). This indicates that priming with oral vitamin D may be necessary to achieve benefit from phototherapy based vitamin D.

Small intestinal bacterial overgrowth (SIBO) is a disorder of excessive bacterial growth in the small intestine, which normally has fewer than 100,000 organisms per millilitre. The small intestine is also the primary site of vitamin B12 absorption. In one study that included dogs in which 22 out of 30 had SIBO, 55\% had low serum vitamin B12 (German, A.J., et al.). In 34 people (mean age 64 years; range 22-95 years) with SIBO there was no difference in serum vitamin B12 between those with increased colonic permeability and asymptomatic individuals.

Interestingly, SIBO was shown to induce vitamin D deficiency as was documented in a case report of a spinal cord injury patient with a sacral pressure sore (Kubota, Yoshitaka, et al.). Therefore it is possible that under certain circumstances vitamin B12 and vitamin D deficiency could be attributable to SIBO.

Salmonella typhimurium produces gastroenteritis in humans and other mammals. It can synthesize vitamin B12, but is actually more virulent when it has lost this ability (Sampson, B A, and E C Gotschlich.).

Consistent with this, mice receiving vitamin D3 supplements experienced reduced severity of Salmonella colitis (Huang, Fu-Chen, and Shun-Chen Huang.).

\section{Tuberculosis}

Mycobacteria, some of which can cause the human disease of tuberculous, are capable of synthesizing vitamin b12 (Minias, Alina, et al.). Interestingly, it appears that the particular Mycobacterial species that cause tuberculosis in people have lost this ability (Kipkorir, Terry, et al.). The role of vitamin B12 in Tuberculosis pathogenesis remains unclear (Gopinath, Krishnamoorthy, et al.). Vitamin B12 regulates Mycobacterial genes that are involved in stress response such as the iniBAC operon (Boot, Maikel, et al.). Comparing patients with tuberculosis meningitis and aseptic meningitis a decreased level of vitamin B12 was found only in patients with tuberculous meningitis whereas unchanged levels were found in patients with aseptic meningitis. Furthermore, ileocaecal tuberculosis is also known to result in vitamin B12 deficiency presumably due to reduced absorption from the intestine (Pannu, Ashok Kumar, and Dinesh Raja Palanisamy).

Despite the role of vitamin b12 in the life cycle of Tuberculosis a study of tuberculosis in Nigerian patients found no difference between serum concentrations of vitamin b12 in tuberculosis vs. asymptomatic patients (Knox-Macaulay H H.). It is possible the inclusion of asymptomatic patients rather than matched controls masked any difference that was present.

Vitamin D deficiency was found in a patient with Mycobacterium szulgai infection (clinically indistinguishable from Mycobacterium tuberculosis), which resolved upon infection treatment (Lotfi, Hadi, et al.). In newly diagnosed sputum positive pulmonary tuberculosis patients, serum vitamin D levels were significantly lower in the tuberculosis group, $(26.7 \pm 1.6 \mathrm{ng} / \mathrm{mL})$ compared to non-TB controls (117.3 $\pm 3.2 \mathrm{ng} / \mathrm{mL}$ ), in some cases (Jaimni, Vijay, et al.), (Sidahmed M.S Elsafi, Samah, et al.). Despite this, a case report of an elderly man noted hypervitaminosis D connected with pulmonary tuberculosis caused by Mycobacterium tuberculosis (Wada, Toshihiro, et al.). Consistent with this low vitamin D levels in type II diabetics was associated with reduced mycobacterial activity (Herrera, María Teresa, et al.). Despite this, immunomodulatory treatments including vitamin D supplementation inhibited growth of clinical multi-drug-resistant tuberculosis strains in human macrophages (Rao Muvva, Jagadeeswara, et al.). Furthermore, clinical trial data indicated that vitamin D supplementation increases the success rate of antituberculosis therapy (Hasanain, Ahmad Farooq, et al.). A 2018 meta-analysis of vitamin D supplementation for pulmonary tuberculosis supports the view that it is effective in combination with other treatments ( $\mathrm{Wu}, \mathrm{Hong}$-xia, et al.). 
The mechanisms of action against Mycobacterium tuberculosis include polarization of monocyte-derived cells expressing LL-37 but not IDO, and cathelicidin downregulation in dendritic cells. (Rao Muvva, Jagadeeswara, et al.), (Rode, Anna K., et al.). Prophylactically, vitamin D supplementation did not result in a lower risk of tuberculosis disease, or acute respiratory infection than placebo among vitamin D-deficient school children in Mongolia (Ganmaa, Davaasambuu, et al.).

Among extrapulmonary tuberculosis cases the mean levels of vitamin $\mathrm{D}$ were also significantly lower than in corresponding controls $(11.9 \pm 8.8$ vs $22.3 \pm 11 \mathrm{ng} / \mathrm{mL} ; \mathrm{p}<0.001)$. Vitamin D deficiency was significantly more frequent among cases $(80 \%$ vs $37.7 \% ; \mathrm{p}<0.001)$, with an odds ratio (OR) of 6.5 (2.5-16) (Hammami, Fatma, et al.).

\section{Leptospira Interrogans}

The role of vitamin B12 in leptospirosis, the disease caused by the spirochete Leptospira interrogans, remains elusive (Murray, Gerald L., et al.). The L. interrogans surface receptor is dispensable for both chronic and acute infections. Despite having a scavenging receptor for vitamin B12 L. interrogans is self-sufficient, being able synthesize vitamin B12 itself in asymptomatic dogs (Kurilung, Alongkorn, et al.). Whether the loss of the ability to synthesize vitamin B12 could cause an increase in the virulence of this spirochete is an open question.

One could speculate if a canine or human played host to a bacterium that had lost the ability to synthesize vitamin B12, and the host itself became deficient in said vitamin, this could trigger a stress response in the bacterium. Depending on the species of bacteria involved this stress could induce a virulence response to gain access to exogenous nutrients.

It is unknown how vitamin D interacts with Leptospira interrogans and vice versa and this will no doubt be a future area of research.

\section{Fungi}

There is very limited literature regarding vitamin B12 and fungi, however some studies exist. A dog with hereditary vitamin B12 deficiency (Imerslund-Gräsbeck syndrome) died of multiorgan failure due to a chronic systemic Scedosporium prolificans infection (Erles, K., et al.). This fungus is multidrug resistant, however it only causes infections opportunistically, such as in the present case of vitamin B12 deficiency.

Similarly another dog was euthanized due to a fungal infection of the liver that caused anorexia, pyrexia and vomiting. Imerslund-Gräsbeck syndrome was identified post-mortem (Kook, P. H., et al.).

There may be some link between atrophic tongue caused by candida species and vitamin B12 deficiency (Terai, Haruhiko, and Masashi Shimahara.). Vitamin B12 deficiency, however, was not found to be a significant risk factor for oral candidiasis (Paillaud, Elena, et al.), (Samaranayake, L.P., and T.W. MacFarlane).

\section{Protozoan}

In broiler chickens infected with Eimeria maxima, a protozoan that causes coccidiosis in poultry, vitamin D supplementation resulted in higher parasite loads and compromised gut architecture (Sakkas, Panagiotis, et al.).

\section{DISCUSSION}

Vitamin B12 and D have diverse effects on microorganisms involved in infection and symbiotic activities as discussed in this review. Of particular interest the activities of both vitamins B12 and D appear to converge upon the Lachnospiraceae family of gut bacteria, suppressing their growth.

The Lachnospiraceae are a family of anaerobic, spore-forming bacteria in the order Clostridiales that are involved in carbohydrate metabolism, including prebiotics, butyrate synthesis, propionate synthesis, 
indole-propionic acid synthesis, $p$-cresol synthesis, and phenol synthesis (Vacca, Mirco, et al.). In particular the genus Lachnospira represents a group of organisms of importance to human health that are negatively correlated with serum levels of both B12 and D. The potential significance of this is discussed. Interestingly, ultra-processed foods have a similar effect on Lachnospira (Cuevas-Sierra, Amanda, et al.).

The Blautia genus is a member of the Lachnospiraceae family which is suppressed by vitamin B12 and vitamin D. Patients with inflammatory bowel disease (IBD) do not absorb vitamin B12 well. Thus high dose supplementation of the vitamin may be required in IBD patients. Under such conditions in mice it was found that Escherichia/ Shigella were abundant and Lactobacillus, Blautia, and Clostridium XVIII genera were at low levels (Zhu, Xuan, et al.).

3-Indolepropionic acid (IPA), or indole-3-propionic acid, is a potent neuroprotective antioxidant that is being studied for therapeutic use in Alzheimer's disease, and is negatively associated with type II diabetes (Chyan, Yau-Jan, et al.), (De Mello, Vanessa D., et al.). IPA is an even more potent scavenger of hydroxyl radicals than melatonin. Within a cohort of 1018 middle-aged women from the TwinsUK cohort, Blautia was the most common taxon associated with lower levels of indole-propionic acid (IPA), indicating that Blautia promoted by vitamins B12 and D is unlikely to protect from Alzheimer's and diabetes (Menni, Cristina, et al.).

Blautia species modify flavonoids which have anti-viral, anticancer, anti-inflammation, antiallergic, antimutagenic, and neuroprotection activities (Burapan, Supawadee, et al.). The consequences of this are not well characterized but it is conceivable that Blautia is involved in the mechanism of action of vitamin D and B12 for and against different microorganisms.

Deficiencies in vitamin B12 and D are common in inflammatory bowel diseases such as Crohn's disease (Gioxari, Aristea, et al.). The butyrate-producing bacterial species Blautia faecis was significantly reduced in Crohn's disease also (Takahashi, Kenichiro, et al.). Butyrate supplementation and perhaps vitamin supplementation could have a role in treating irritable bowel diseases, inflammatory processes, atherosclerosis, and maturation of the immune system (Sitkin, Stanislav, et al.), (Vacca, Mirco, et al.).

\section{REFERENCE}

Arumugam, Manimozhiyan, et al. "Enterotypes of the Human Gut Microbiome.” Nature, vol. 473, no. 7346, 2011, pp. 174-180., doi:10.1038/nature09944.

Boot, Maikel, et al. "IniBAC Induction Is Vitamin b12- AND Mutab-Dependent IN Mycobacterium Marinum.” Journal of Biological Chemistry, vol. 291, no. 38, 2016, pp. 19800-19812., doi:10.1074/jbc.m116.724088.

Burapan, Supawadee, et al. "Demethylation of Polymethoxyflavones by Human Gut Bacterium, Blautia Sp. MRGPMF1.” J Agric Food Chem, vol. 65, no. 8, 1 Mar. 2017, pp. 1620-1629, doi:10.1021/acs.jafc.7b00408.s001.

Charoenngam, Nipith, et al. "The Effect of Various Doses of Oral Vitamin d3 Supplementation on Gut Microbiota in Healthy Adults: A Randomized, Double-Blinded, Dose-Response Study.” Anticancer Research, vol. 40, no. 1, 2019, pp. 551-556., doi:10.21873/anticanres.13984.

Chatterjee, Ishita, et al. "Vitamin D Receptor Promotes Healthy Microbial Metabolites and Microbiome." Scientific Reports, vol. 10, no. 1, 2020, doi:10.1038/s41598-020-64226-7.

Chen, Po-Jung, et al. "Daily Full Spectrum Light Exposure Prevents Food Allergy-like Allergic Diarrhea by Modulating Vitamin d3 and Microbiota Composition." Npj Biofilms and Microbiomes, vol. 7, no. 1, 2021, doi:10.1038/s41522-021-00213-8.

Chyan, Yau-Jan, et al. "Potent Neuroprotective Properties against the Alzheimer $\beta$-Amyloid by an Endogenous Melatonin-Related Indole Structure, Indole-3-Propionic Acid.” Journal of Biological Chemistry, vol. 274 , no. 31, 1999, pp. 21937-21942., doi:10.1074/jbc.274.31.21937.

Cordonnier, Charlotte, et al. "Vitamin b12 Uptake by the Gut Commensal Bacteria Bacteroides Thetaiotaomicron Limits the Production of Shiga Toxin by Enterohemorrhagic Escherichia Coli." Toxins, vol. 8, no. 1, 2016, p. 14., doi:10.3390/toxins8010014.

Cuevas-Sierra, Amanda, et al. "Gut Microbiota Differences According to Ultra-Processed Food Consumption in a Spanish Population.” Nutrients, vol. 13, no. 8, 2021, p. 2710., doi:10.3390/nu13082710.

De Mello, Vanessa D., et al. "Indolepropionic Acid and Novel Lipid Metabolites Are Associated with a Lower Risk of Type 2 Diabetes in the Finnish Diabetes Prevention Study." Scientific Reports, vol. 7, no. 1, 2017, doi:10.1038/srep46337. 
Erles, K., et al. "Systemic Scedosporium Prolificans infection in an 11-Month-Old Border collie with Cobalamin Deficiency Secondary to Selective Cobalamin Malabsorption (canine Imerslund-Gräsbeck syndrome)." Journal of Small Animal Practice, vol. 59, no. 4, 2017, pp. 253-256., doi:10.1111/jsap.12678.

Ganmaa, Davaasambuu, et al. "Vitamin D Supplements for Prevention of Tuberculosis Infection and Disease." New England Journal of Medicine, vol. 383, no. 4, 2020, pp. 359-368., doi:10.1056/nejmoa1915176.

German, A.J., et al. "Comparison of Direct and Indirect Tests for Small Intestinal Bacterial Overgrowth and AntibioticResponsive Diarrhea in Dogs.” Journal of Veterinary Internal Medicine, vol. 17, no. 1, 2003, pp. 33-43., doi:10.1111/j.1939-1676.2003.tb01321.x.

Gioxari, Aristea, et al. "Serum Vitamins D, B9 and B12 in Greek Patients with Inflammatory Bowel Diseases." Nutrients, vol. 12, no. 12, 2020, p. 3734., doi:10.3390/nu12123734.

Gokhale, Sucheta, and Anirban Bhaduri. "Provitamin d3 Modulation through Prebiotics Supplementation: Simulation Based Assessment." Scientific Reports, vol. 9, no. 1, 17 Dec. 2019, p. 19267., doi:10.1038/s41598-019-556992.

Gopinath, Krishnamoorthy, et al. "Vitamin B12 Metabolism in Mycobacterium Tuberculosis." Future Microbiology, vol. 8, no. 11, 2013, pp. 1405-1418., doi:10.2217/fmb.13.113.

Grützner, Niels, et al. "Serum Folate, Cobalamin, Homocysteine and Methylmalonic Acid Concentrations in Pigs with Acute, Chronic or Subclinical Lawsonia Intracellularis Infection.” The Veterinary Journal, vol. 203, no. 3, 2015, pp. 320-325., doi:10.1016/j.tvj1.2014.12.022.

Hammami, Fatma, et al. "The Association between Vitamin D Deficiency and Extrapulmonary Tuberculosis: CaseControl Study." Tuberculosis, vol. 126, 2021, p. 102034., doi:10.1016/j.tube.2020.102034.

Hasanain, Ahmad Farooq, et al. "Efficacy and Safety of Cholecalciferol-Augmented Anti-Tuberculosis Therapy for Treatment of Naïve Patients with Pulmonary Tuberculosis: A Randomized, Controlled, Clinical Study." Indian Journal of Tuberculosis, vol. 66, no. 1, 2019, pp. 111-117., doi:10.1016/j.ijtb.2018.06.004.

Herrera, María Teresa, et al. "Low Serum Vitamin D Levels in Type 2 Diabetes Patients Are Associated with Decreased Mycobacterial Activity." BMC Infectious Diseases, vol. 17, no. 1, 2017, doi:10.1186/s12879-017-2705-1.

Huang, Fu-Chen, and Shun-Chen Huang. "Active Vitamin d3 Attenuates the Severity of Salmonella Colitis in Mice by Orchestrating Innate Immunity." Immunity, Inflammation and Disease, vol. 9, no. 2, 2021, pp. 481-491., doi:10.1002/iid3.408.

Ikezaki, Hiroaki, et al. "Higher Dietary Cholesterol AND $\Omega-3$ Fatty Acid Intakes Are Associated with a Lower Success Rate of Helicobacter PYLORI Eradication Therapy in Japan.” The American Journal of Clinical Nutrition, vol. 106, no. 2, 2017, pp. 581-588., doi:10.3945/ajcn.116.144873.

Jaimni, Vijay, et al. “Association of Vitamin D Deficiency and Newly Diagnosed Pulmonary Tuberculosis.” Pulmonary Medicine, vol. 2021, 2021, pp. 1-6., doi:10.1155/2021/5285841.

Kipkorir, Terry, et al. "De Novo Cobalamin BIOSYNTHESIS, Transport, and Assimilation and Cobalamin-Mediated Regulation of Methionine Biosynthesis IN Mycobacterium Smegmatis.” Journal of Bacteriology, vol. 203, no. 7, 2021, doi:10.1128/jb.00620-20.

Knox-Macaulay H H. Serum cobalamin concentration in tuberculosis: a study in the Guinea savanna of Nigeria. Trop Geogr Med, 42: 1990, pp. 146-150.

Kook, P. H., et al. "Hepatic Fungal Infection in a Young Beagle with Unrecognised Hereditary Cobalamin Deficiency (Imerslund-Gräsbeck Syndrome)." Journal of Small Animal Practice, vol. 56, no. 2, 2014, pp. 138-141., doi:10.1111/jsap.12251.

Kubota, Yoshitaka, et al. "Small Intestinal Bacterial Overgrowth as a Cause of Protracted Wound Healing and Vitamin d Deficiency in a Spinal Cord Injured Patient with a Sacral Pressure Sore: A Case Report." BMC Gastroenterology, vol. 20, no. 1, 2020, doi:10.1186/s12876-020-01423-8.

Kurilung, Alongkorn, et al. "Chronology of Emergence of the Genus Leptospira and over-Representation of Gene Families Enriched by vitamin B2, B12 Biosynthesis, Cell Adhesion and External Encapsulating Structure in L. Interrogans Isolates from Asymptomatic Dogs." Infection, Genetics and Evolution, vol. 73, 2019, pp. 712., doi:10.1016/j.meegid.2019.04.005.

Lotfi, Hadi, et al. "Mycobacterium Szulgai Pulmonary Infection in a Vitamin d-Deficient Patient: A Case Report." Clinical Case Reports, vol. 9, no. 3, 2021, pp. 1146-1149., doi:10.1002/ccr3.3692.

Luthold, Renata V., et al. "Gut Microbiota Interactions with the Immunomodulatory Role of Vitamin d in Normal Individuals.” Metabolism, vol. 69, 2017, pp. 76-86., doi:10.1016/j.metabol.2017.01.007.

Matsumoto, Natsuko, et al. "Relationship between Nutrient Intake and Human Gut Microbiota in Monozygotic Twins." Medicina, vol. 57, no. 3, 2021, p. 275., doi:10.3390/medicina57030275.

Minias, Alina, et al. "Cobalamin Is Present in Cells of Non-Tuberculous Mycobacteria, but Not IN Mycobacterium Tuberculosis." Scientific Reports, vol. 11, no. 1, 2021, doi:10.1038/s41598-021-91430-w.

Missailidis, Catharina, et al. "Vitamin D and Phenylbutyrate Supplementation Does Not Modulate Gut Derived Immune Activation in HIV-1.” Nutrients, vol. 11, no. 7, 2019, p. 1675., doi:10.3390/nu11071675.

Murray, Gerald L., et al. "Major Surface Protein lip132 Is Not Required for Either Acute or Chronic Infection with Leptospira Interrogans." Infection and Immunity, vol. 77, no. 3, 2009, pp. 952-958., doi:10.1128/iai.01370-08. 
Nadeau É., et al. "Administration of Pediococcus Acidilactici or Saccharomyces Cerevisiae Boulardii Modulates Development of Porcine Mucosal Immunity and Reduces Intestinal Bacterial Translocation after Escherichia Coli Challenge.” Journal of Animal Science, vol. 87, no. 3, 2009, pp. 922-934., doi:10.2527/jas.2008-0919.

Nasri, Hamid, and Azar Baradaran. "The Influence of Serum 25-Hydroxy Vitamin D Levels on Helicobacter Pylori Infections in Patients with End-Stage Renal Failure on Regular Hemodialysis.” Saudi Journal of Kidney Diseases and Transplantation, vol. 18, no.2, June 2007, pp.215-219. https://www.sjkdt.org/text.asp?2007/18/2/215/32312

Ooi, Jot Hui, et al. "Vitamin d Regulates the Gut Microbiome and Protects Mice from Dextran Sodium Sulfate-Induced Colitis." The Journal of Nutrition, vol. 143, no. 10, 2013, pp. 1679-1686., doi:10.3945/jn.113.180794.

Paillaud, Elena, et al. "Oral Candidiasis and Nutritional Deficiencies in Elderly Hospitalised Patients." British Journal of Nutrition, vol. 92, no. 5, 2004, pp. 861-867., doi:10.1079/bjn20041264.

Pannu, Ashok Kumar, and Dinesh Raja Palanisamy. "Ileocaecal Tuberculosis: An under-Recognised Cause of Vitamin b12 Deficiency." Tropical Doctor, vol. 49, no. 2, 2018, pp. 143-144., doi:10.1177/0049475518816590.

Qureshi, G.Ali, et al. "The Neurochemical Markers in Cerebrospinal Fluid to Differentiate between Aseptic and Tuberculous Meningitis." Neurochemistry International, vol. 32, no. 2, 1998, pp. 197-203., doi:10.1016/s01970186(97)00061-2.

Rabbone, Ivana, et al. "Microbiota, Epidemiological and Nutritional Factors Related to Ketoacidosis at the Onset of Type 1 Diabetes.” Acta Diabetologica, vol. 57, no. 11, 2020, pp. 1337-1349., doi:10.1007/s00592-020-01555z.

Rao Muvva, Jagadeeswara, et al. "Immunomodulatory Agents Combat Multidrug-Resistant Tuberculosis by IMPROVING ANTIMICROBIAL IMMUNITY.” The Journal of Infectious Diseases, vol. 224, no. 2, 2021, pp. 332-344., doi:10.1093/infdis/jiab100.

Rao Muvva, Jagadeeswara, et al. "Polarization of Human Monocyte-Derived Cells with Vitamin d Promotes Control of Mycobacterium Tuberculosis Infection." Frontiers in Immunology, vol. 10, 2020, doi:10.3389/fimmu.2019.03157.

Riordan, S. M., et al. "Luminal Bacteria and Small-Intestinal Permeability.” Scandinavian Journal of Gastroenterology, vol. 32, no. 6, 1997, pp. 556-563., doi:10.3109/00365529709025099.

Robles-Vera, Iñaki, et al. "Impact of Vitamin D Deficit on the Rat Gut Microbiome.” Nutrients, vol. 11, no. 11, 2019, p. 2564., doi:10.3390/nu11112564.

Rode, Anna K., et al. "Vitamin D Counteracts MYCOBACTERIUM Tuberculosis-Induced CATHELICIDIN Downregulation IN Dendritic Cells and Allows Th1 Differentiation AND IFN $\gamma$ Secretion." Frontiers in Immunology, vol. 8, 2017, doi:10.3389/fimmu.2017.00656.

Sakkas, Panagiotis, et al. "Dietary Vitamin d Improves Performance and Bone Mineralisation, but Increases Parasite Replication and Compromises Gut Health IN Eimeria-Infected Broilers.” British Journal of Nutrition, vol. 122, no. 6, 2019, pp. 676-688., doi:10.1017/s0007114519001375.

Samaranayake, L.P., and T.W. MacFarlane. "A Retrospective Study of Patients with Recurrent Chronic Atrophic Candidosis.” Oral Surgery, Oral Medicine, Oral Pathology, vol. 52, no. 2, 1981, pp. 150-153., doi:10.1016/00304220(81)90312-1.

Sampson, B A, and E C Gotschlich. "Elimination of the Vitamin B12 Uptake or Synthesis Pathway Does Not Diminish the Virulence of Escherichia Coli K1 or Salmonella Typhimurium in Three Model Systems." Infection and Immunity, vol. 60, no. 9, 1992, pp. 3518-3522., doi:10.1128/iai.60.9.3518-3522.1992.

Shafrir, Asher, et al. "The Association between Serum Vitamin D Levels and Helicobacter Pylori Presence and Eradication." Nutrients, vol. 13, no. 1, 2021, p. 278., doi:10.3390/nu13010278.

Shieh, Albert, et al. "Pilot Trial of Vitamin d3 and Calcifediol in Healthy Vitamin d Deficient Adults: Does It Change the Fecal Microbiome?" The Journal of Clinical Endocrinology \& Metabolism, 2021, doi:10.1210/clinem/dgab573.

Sidahmed M.S Elsafi, Samah, et al. "Vitamin D Level and It Is Association with the Severity of Pulmonary Tuberculosis in Patients Attended to Kosti Teaching Hospital, Sudan.” AIMS Microbiology, vol. 6, no. 1, 2020, pp. 65-74., doi:10.3934/microbiol.2020004.

Sitkin, Stanislav, et al. "How to Increase the Butyrate-Producing Capacity of the Gut Microbiome: Do Ibd Patients Really Need Butyrate Replacement and Butyrogenic Therapy?” Journal of Crohn's and Colitis, vol. 12, no. 7, 2018, pp. 881-882., doi:10.1093/ecco-jcc/jjy033.

Soltys, Katarina, et al. "Seasonal Changes of Circulating 25-Hydroxyvitamin D Correlate with the Lower Gut Microbiome Composition in Inflammatory Bowel Disease Patients." Scientific Reports, vol. 10, no. 1, 2020, doi:10.1038/s41598-020-62811-4.

Takahashi, Kenichiro, et al. "Reduced Abundance of Butyrate-Producing Bacteria Species in the Fecal Microbial Community in Crohn's Disease.” Digestion, vol. 93, no. 1, 2016, pp. 59-65., doi:10.1159/000441768.

Terai, Haruhiko, and Masashi Shimahara. "Atrophic Tongue Associated with Candida.” Journal of Oral Pathology and Medicine, vol. 34, no. 7, 2005, pp. 397-400., doi:10.1111/j.1600-0714.2005.00324.x.

Vacca, Mirco, et al. "The Controversial Role of Human Gut Lachnospiraceae.” Microorganisms, vol. 8, no. 4, 2020 , p. 573., doi:10.3390/microorganisms8040573. 
Wada, Toshihiro, et al. "Acute Hypercalcemia and Hypervitaminosis d Associated with Pulmonary Tuberculosis in an Elderly Patient : A Case Report and Review of the Literature.” The Journal of Medical Investigation, vol. 66, no. 3.4, 2019, pp. 351-354., doi:10.2152/jmi.66.351.

Wang, Jun, et al. "Genome-Wide Association Analysis Identifies Variation in Vitamin d Receptor and Other Host Factors Influencing the Gut Microbiota." Nature Genetics, vol. 48, no. 11, 2016, pp. 1396-1406., doi:10.1038/ng.3695.

Wang, Xin, et al. "Vitamin d Decreases Plasma Trimethylamine-n-Oxide Level in Mice by Regulating Gut Microbiota." BioMed Research International, vol. 2020, 2020, pp. 1-11., doi:10.1155/2020/9896743.

Waterhouse, Mary, et al. "Vitamin D and the Gut Microbiome: A Systematic Review of in Vivo Studies." European Journal of Nutrition, vol. 58, no. 7, Oct. 2019, pp. 2895-2910., doi:10.1007/s00394-018-1842-7.

Wu, G. D., et al. "Linking Long-Term Dietary Patterns with Gut Microbial Enterotypes." Science, vol. 334, no. 6052, 2011, pp. 105-108., doi:10.1126/science.1208344.

Wu, Hong-xia, et al. "Effects of Vitamin d Supplementation on the Outcomes of Patients with Pulmonary Tuberculosis: A Systematic Review and Meta-Analysis." BMC Pulmonary Medicine, vol. 18, no. 1, 2018, doi:10.1186/s12890-018-0677-6.

Zhang, Yong-Guo, et al. "Vitamin D Receptor Protects Against dysbiosis and Tumorigenesis via the JAK/STAT Pathway In Intestine." Cellular and Molecular Gastroenterology and Hepatology, vol. 10, no. 4, 2020, pp. 729746., doi:10.1016/j.jcmgh.2020.05.010.

Zhou, Anni, et al. "Vitamin D3 Inhibits Helicobacter Pylori Infection by Activating the Vitd3/Vdr-Camp Pathway in Mice." Frontiers in Cellular and Infection Microbiology, vol. 10, 2020, doi:10.3389/fcimb.2020.566730.

Zhou, Xueyan, et al. "Effect and Mechanism of Vitamin d on the Development of Colorectal Cancer Based on Intestinal Flora Disorder." Journal of Gastroenterology and Hepatology, vol. 35, no. 6, 2019, pp. 1023-1031., doi:10.1111/jgh.14949.

Zhu, Xuan, et al. "Impact of Cyanocobalamin and Methylcobalamin on Inflammatory Bowel Disease and the Intestinal Microbiota Composition." J Agric Food Chem., vol. 67, no. 3, 23 Jan. 2019, pp. 916-926., doi:10.1021/acs.jafc.8b05730.s002.

Zuo, Kun, et al. "Dysbiotic Gut Microbes May Contribute to Hypertension by Limiting Vitamin d Production." Clinical Cardiology, vol. 42, no. 8, 2019, pp. 710-719., doi:10.1002/clc.23195.

\section{Appendix}

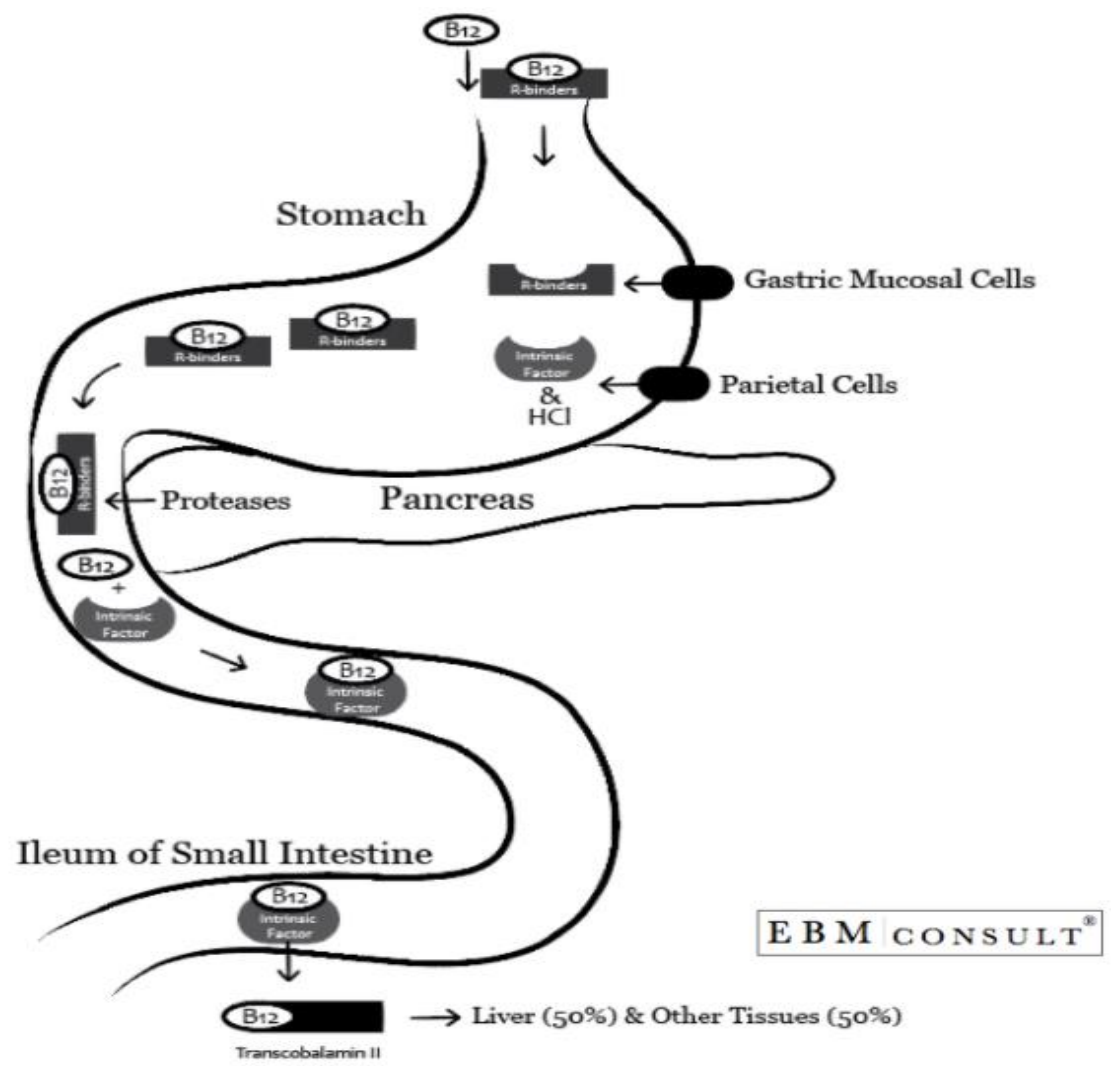

Figure 1: Vitamin B12 absorption. Reproduced without permission:

*Source: https://www.ebmconsult.com/articles/vitamin-B12-absorption-mechanism-intestine-intrinsic-factor $--0--$ 